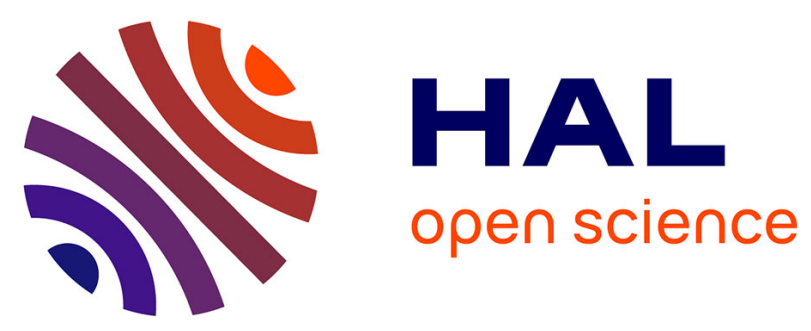

\title{
Reducing the number of experiments required for modeling the hydrocracking process with kriging through Bayesian transfer learning
}

\author{
Loïc Iapteff, Julien Jacques, Matthieu Rolland, Benoît Celse
}

\section{- To cite this version:}

Loïc Iapteff, Julien Jacques, Matthieu Rolland, Benoit Celse. Reducing the number of experiments required for modeling the hydrocracking process with kriging through Bayesian transfer learning. Journal of the Royal Statistical Society: Series C Applied Statistics, 2021, 70 (5), pp.1344-1364. $10.1111 /$ rssc.12516 . hal-02935247v2

HAL Id: hal-02935247

https://hal.science/hal-02935247v2

Submitted on 23 Nov 2021

HAL is a multi-disciplinary open access archive for the deposit and dissemination of scientific research documents, whether they are published or not. The documents may come from teaching and research institutions in France or abroad, or from public or private research centers.
L'archive ouverte pluridisciplinaire HAL, est destinée au dépôt et à la diffusion de documents scientifiques de niveau recherche, publiés ou non, émanant des établissements d'enseignement et de recherche français ou étrangers, des laboratoires publics ou privés. 


\title{
Reducing the number of experiments required for model- ing the hydrocracking process with kriging through Bayesian transfer learning
}

\author{
lapteff Loïc ${ }^{a, b}$, Jacques Julien ${ }^{b}$, Rolland Matthieu $^{a}$, Celse Benoit ${ }^{a}$ \\ ${ }^{a}$ IFPEN, Lyon, France. \\ ${ }^{b}$ Laboratoire ERIC, Lyon, France. \\ E-mail: loic.iapteff@ifpen.fr
}

\begin{abstract}
Summary. The objective is to improve the learning of a regression model of the hydrocracking process using a reduced number of observations. When a new catalyst is used for the hydrocracking process, a new model must be fitted. Generating new data is expensive and therefore it is advantageous to limit the amount of new data generation. Our idea is to use a second data set of measurements made on a process using an old catalyst. This second data set is large enough to fit performing models for the old catalyst. In this work, we use the knowledge from this old catalyst to learn a model on the new catalyst. This task is a transfer learning task. We show that the results are greatly improved with a Bayesian approach to transfer linear model and kriging model.

Keywords: Transfer Knowledge of Parameters, Regression modeling, Gaussian Process, MCMC algorithm, kriging
\end{abstract}

\section{Introduction}

\subsection{Industrial Challenge}

Refineries convert crude oil into usable products, mostly fuels for the transport industry like gasoline, kerosene for planes or diesel, and high purity chemicals that will be used to produce plastics including propylene, butadiene and aromatics. The most important unit operations are distillations to separate the chemical constituents according to their boiling points, and two types of chemical reaction: 1) purification that aims at removing impurities like sulfur, nitrogen, and 2) conversion that changes the chemical structure of the molecules and improve their commercial value. A refinery is thus a complex network of interconnected units. A modern refinery costs in the order of several billion euros, and there is a great financial interest to optimize design and operation.

Reactions take place in presence of a catalyst whose aim is to reduce temperature, pressure and time required to achieve the performance as well as to orientate the reactions toward the desired products (selectivity). For each reaction, several vendors offer their own catalysts. When offering a new catalyst, a vendor must guarantee its performance: a lot of effort of the catalyst development cycle is to be able to predict, as accurately as possible, its performance in the customer's refinery. Performance prediction is based on both experimental data and models. Experiments are very expensive as they must be performed in conditions mimicking those of refineries on a variety of feedstocks that are not commercial and difficult to obtain. The cost for one point is around 10,000 
euros. Modeling can be based on either physical approaches or statistical approaches. Physical approaches are possible for simple molecules and when the scientific knowledge is sufficient enough to predict all properties of interest from the chemical composition. In most cases, this is not possible and correlations are built and used. New catalysts are constantly being developed so that each new generation of a catalyst requires a new model that is built from scratch from new experiments. In this paper, we are interested in using previous knowledge on an old catalyst to build a model for a new catalyst with fewer new data points.

This work focuses on the hydrocracking process that converts heavy products from vacuum distillation into diesel and lubricants, which are more valuable products. Chemically speaking, it breaks long hydrocarbon chains into smaller bits in the presence of high temperature $\left(>380^{\circ} \mathrm{C}\right)$ and hydrogen under high pressure $(\sim 150$ bars $)$. Downstream of the hydrocracking reactor, a distillation column splits the products into several cuts, one being the diesel cut. More specifically, we want to predict the density of the diesel cut based on information about the feedstock, the operating conditions and some information on the diesel cut.

The challenge in this paper is to build the best predictive model for a new catalyst with the fewest observations on that new catalyst ("new") and having access to many observations on a previous generation catalyst ("old"). In the field of catalyst models, when defining a model for a new catalyst, data related to the previous catalysts are never used. In this paper, we propose an approach that greatly reduces the number of observations required to build an efficient kriging model on the new catalyst, and therefore the cost of fitting this new model. For this reason, we will be working on two data sets, one corresponding to the old catalyst, large in size, and the other corresponding to a new generation of catalyst. We want the latter to be as small as possible. This task is known as transfer learning (Pan and Yang (2010)).

\subsection{Related work on Transfer Learning}

Let's define a domain as $\mathcal{D}=(\mathcal{X}, P(\mathcal{X}))$ with $\mathcal{X}$ a feature space and $P(\mathcal{X})$ its probability distribution, and an associated task $\mathcal{T}=(Y, f)$ with $f$ the function used to predict $y \in Y$ given $\boldsymbol{x} \in \mathcal{X}$. We name $\mathcal{D}_{s}$ and $\mathcal{T}_{s}$ the domain and task of source data and $\mathcal{D}_{t}$ and $\mathcal{T}_{t}$ the domain and task of the target data. There are two distinct areas in Transfer Learning, the Transductive Transfer Learning when $\mathcal{D}_{s} \neq \mathcal{D}_{t}$ and the Inductive Transfer Learning when $\mathcal{T}_{s} \neq \mathcal{T}_{t}$. For this work, the source data are those from the old catalyst, while the target data are those from the new one. $\mathcal{X}_{s}=\mathcal{X}_{t}$ is the space of the 12 features previously presented while $Y_{s}=Y_{t}$ is DIES_D154, the variable to be predicted. The values of these 12 features are determined by the refiner, and we can therefore assume that $P\left(\mathcal{X}_{s}\right)=P\left(\mathcal{X}_{t}\right)$. Thus, $\mathcal{D}_{s}=\mathcal{D}_{t}$ and $\mathcal{T}_{s} \neq \mathcal{T}_{t}$ which means it is an Inductive Transfer Learning problem.

Inductive Transfer Learning is classically split into three categories: transfer knowledge of instances, transfer of features representation and transfer of parameters. The distinction is, as their name suggests, the way in which information is transferred. Literature on transfer learning is abundant and we will only present some of them here. Those interested can read reviews (Pan and Yang (2010) and Tsung et al. (2018)), the latter presenting a more statistical point of view. 
The transfer knowledge of instances is based on the direct reuse of observations from the source data set. To quote some works of this method, we can mention Transfer Adaptive Boosting (Dai et al. (2007)), which adapts the Adaptive Boosting algorithm (AdaBoost) for Transfer Learning in classification or its adaptation for regression (Pardoe and P. Stone (2010)). The AdaBoost algorithm builds a set of weak learners iteratively, a weak learner being a poor predictor or classifier, barely better than chance but robust to overfitting. The observations are weighted and the first weak learner is learned on the observations having all the same weight. The weak learners are then successively learned by increasing the weights of the observations incorrectly predicted by the previous learner and decreasing the weights of those well predicted. The prediction is then made by averaging the results of the different learners. For the Transfer AdaBoost algorithm, both source and target data are used. For the target data, the evolution of the weights at each iteration is the same as for AdaBoost. Conversely, for the source data, the weight is increased if the observation is well predicted and therefore considered useful for learning, and it is otherwise decreased. At each iteration, the learning is performed on the source and target data but the error is calculated on the target data only. Another work on instance transfer uses a method that generates more target data (Salaken et al. (2019)). The algorithm produces new target observations and can be used when $\mathcal{X}_{s}=\mathcal{X}_{t}$. Source domain is abstracted using autoencoder to obtain a new domain with the same number of features as the target domain. Then, clustering is performed on the source domain to have as many clusters as the target data. Each cluster center is associated with a target point and the distribution of data around these centers is reapplied around the associated target point.

For transfer knowledge of features representation, the problem is similar to multitask learning, where the aim is to find the common feature representation (Argyriou, Evgeniou, and Pontil (2007); Argyriou, Pontil, et al. (2008)), which can be applied for transfer learning. As it looks for a low dimensional representation shared by the different tasks, this method is more useful when the number of features is large. The proposed algorithm by Argyriou, Evgeniou, and Pontil (2007); Argyriou, Pontil, et al. (2008) works in two alternating steps. The first independently learn parameters of different tasks models. The second aims at keeping the tasks coupled by learning common features across the tasks in an unsupervised way.

The third method is the transfer knowledge of parameters. For Random Forest models, two algorithms can be cited (Segev et al. (2016)): Structure Expansion Reduction (SER) and Structure Transfer (STRUT). They both transform the forest learned on source data, tree by tree. SER is a two step algorithm: expansion and reduction. Expansion extends the tree by constructing new sub-trees starting from leaves with target data that reached them. Reduction runs through all internal nodes and transforms them into a leaf if the error is reduced compared to the empirical error of its sub-tree. The STRUT algorithm works on the thresholds by readjusting them on the target data, keeping the feature on which the separation is made. For linear models, one possibility is to find a link between the source and the target model by assuming that some parameters are unchanged or subject to the same transformation (Bouveyron and Jacques (2010)). This makes it possible to reduce the number of parameters to be estimated and maintains knowledge of the source data. Another approach has been proposed to transfer knowl- 
edge of parameters in Support Vector Machine models (Evgeniou and Pontil (2004)). The idea is to assume that the weighted vector of source and target tasks can be decomposed into two terms, one common to both, the other different.

Independently from the type of model, Bayesian approaches are often considered for transfer knowledge of parameters. For instance, Raina, Ng, and Koller (2006) constructs a Gaussian informative prior to the document classification task using logistic regression. They construct a non-diagonal covariance matrix for the Gaussian prior using auxiliary document classification tasks. The objective is to find the correlated words and thus the correlated parameters instead of assuming their independence. Still with a Bayesian approach, Launay, Philippe, and Lamarche (2015) use a hierarchical Bayesian model to forecast electricity load using a small data set. The prior distributions are constructed using another data set, which is larger, on which the model is known to be effective. The parametric regression model is non-linear and a Gaussian prior is chosen. Another paper proposes to solve regression problems in an adaptive way via the Gaussian Process (B. Cao et al. (2010)). It assumes that the two Gaussian Process regression models for the source and target tasks share the same parameters $\boldsymbol{\theta}$ in their kernel functions. The dissimilarity between source and target task is represented by a parameter that follows a Gamma distribution and thus the model automatically learns the similarity between the tasks. The covariance function therefore differs by a coefficient depending on whether the observations come from the same task or not. This approach, which will be named TGP, is designed for single source problem and Wei et al. (2017) adapt it to the case of multi-source transfer. They first propose an approach to express the similarity between different source set with the target set as in TGP. For this approach, the covariance function differs by a different coefficient for each pairs of source target set. They show that these coefficients must be equal for the covariance matrix to be positive semi-definite, and is therefore not suitable because it does not represent the different similarities between the different source target pairs. They then propose a stacking method by building a TGP for each source target pairs and combining their predictions, and show the superiority of this approach compare to other multi source transfer problems. Da et al. (2019) propose a method to speed up the learning, by partitioning the source data set in multiple subsets. Then, for each subset coupled with the full target data set, TGP are fitted in parallel and sharing the same parameters. Still concerning the Gaussian Processes, Liu, Cai, and Ong (2018) performs a state of the art on Multi Output Gaussian Processes, and thus on the asymmetric case which corresponds to transfer learning. For this asymmetric case, they focus on the multi-fidelity output scenario.

As our features are already selected, the transfer knowledge of feature representation method is not suitable. We opted for transfer knowledge of model parameters as we consider it to be well suited to the transformation of models that have proven efficient on the source data.

\subsection{Our data sets}

The data sets are composed of data originating from plants in the form of time series, with measurements being performed daily. For the "old" catalyst set, the data came from 8 plants for a total of 3,177 observations. For the "new" catalyst, the data came 
Table 1. Description of the features used to learn the model. The last line describes the variable to be predicted.

\begin{tabular}{|c|c|}
\hline Feature & Description \\
\hline PPH2 & $\begin{array}{l}\text { Hydrogen (H2) partial pressure. Hydrogen is used to break molecules } \\
\text { bonds. }\end{array}$ \\
\hline TWABT & $\begin{array}{l}\text { Average temperature of hydrocracking reactor (WABT: Weight Average } \\
\text { Bed temperature). }\end{array}$ \\
\hline FEED_D154 & $\begin{array}{l}\text { Feedstock relative density measured at } 15^{\circ} \mathrm{C} \text { with respect to density of } \\
\text { water at } 4{ }^{\circ} \mathrm{C} \text {. }\end{array}$ \\
\hline $\begin{array}{l}\text { FEED_DS05 } \\
\text { FEED_DS50 } \\
\text { FEED_DS95 }\end{array}$ & $\begin{array}{l}\text { Temperature at which a given percentage of the feedstock is evaporated. } \\
\text { Simulated distillation (DS) is an indirect measurement of the distillation } \\
\text { temperatures based on gas phase chromatography. It produces a curve } \\
\text { that we reduce to } 3 \text { temperatures at } 5,50 \text { and } 95 \% \text { of evaporation. }\end{array}$ \\
\hline FEED_NIT & $\begin{array}{l}\text { Nitrogen content in feedstock. Nitrogen is a catalyst inhibitor found in } \\
\text { crude oil. }\end{array}$ \\
\hline FEED_SULF & $\begin{array}{l}\text { Sulfur content in feedstock. Sulfur is a pollutant causing acid rains after } \\
\text { its combustion in engines. }\end{array}$ \\
\hline $\begin{array}{l}\text { DIES_DS05 } \\
\text { DIES_DS50 } \\
\text { DIES_DS95 }\end{array}$ & $\begin{array}{l}\text { Temperature at which } 5,50 \text { and } 95 \% \text { of the diesel output is evaporated } \\
\text { (see FEED_DS for details). }\end{array}$ \\
\hline $\mathrm{X} 370+$ & $\begin{array}{l}\text { Percentage of conversion of the feedstock } 370+\text { cut. It indicates how } \\
\text { much of hydrocracking was performed. }\end{array}$ \\
\hline $\begin{array}{l}\text { DIES_D154 } \\
\text { (To predict) }\end{array}$ & $\begin{array}{l}\text { Relative density of diesel measured at } 15^{\circ} \mathrm{C} \text { with respect to density of } \\
\text { water at } 4^{\circ} \mathrm{C} \text {. The feature to be predicted. }\end{array}$ \\
\hline
\end{tabular}

from 2 plants for a total of 1,004 observations.

Experts selected 12 features presented in Table 1 that are defined bellow, starting from the top. The first two features are process parameters: partial pressure of hydrogen (PPH2) and temperature (WABT). Next are four properties of the feedstock, namely density (FEED_D154) and temperatures for which 5, 50 and $95 \%$ of the feedstocks are evaporated (FEED_DS05, 50 and 95). Each refinery has its own definition for the diesel distillation cut, some cut higher and some lower, which simply means that the distillation temperatures are different. This, of course, has an impact of the diesel density (a higher cut diesel has more molecules that vaporizes at high temperature and has thus a higher density). Therefore, information on the diesel distillation is included in the model through the DS features (DIES_DS05, 50 and 95). The last feature is the conversion $(\mathrm{X} 370+)$ which is the fraction of the heavy products that have been hydrocracked. Conversion is, of course, a consequence of the process parameters and feedstock properties. Its prediction is modeling work in itself (see for example Becker, Celse, et al. (2016)). All of those features are quantitative variables, including the output to be predicted, and we want to build a regression model:

$$
y_{i}=f\left(\boldsymbol{x}_{i}\right)+\epsilon_{i}
$$

where $y_{i}$ is the diesel density (DIES_D154) and $\boldsymbol{x}_{i}=\left(x_{i, 1}, \ldots, x_{i, 12}\right)$ is the vector of the 12 features presented above for observation i. Observations are here defined as data points from plant operation track logs and are a series of successive observations 
labeled by date. The temporal aspect is not considered and each point is assumed to be independent of the others.

The two data sets used in this study are different enough that the feature correlation matrix are different for the two catalysts (Figure 1). Correlations between input features and the property to be predicted (DIES_D154) are slightly distinct. For the old catalyst
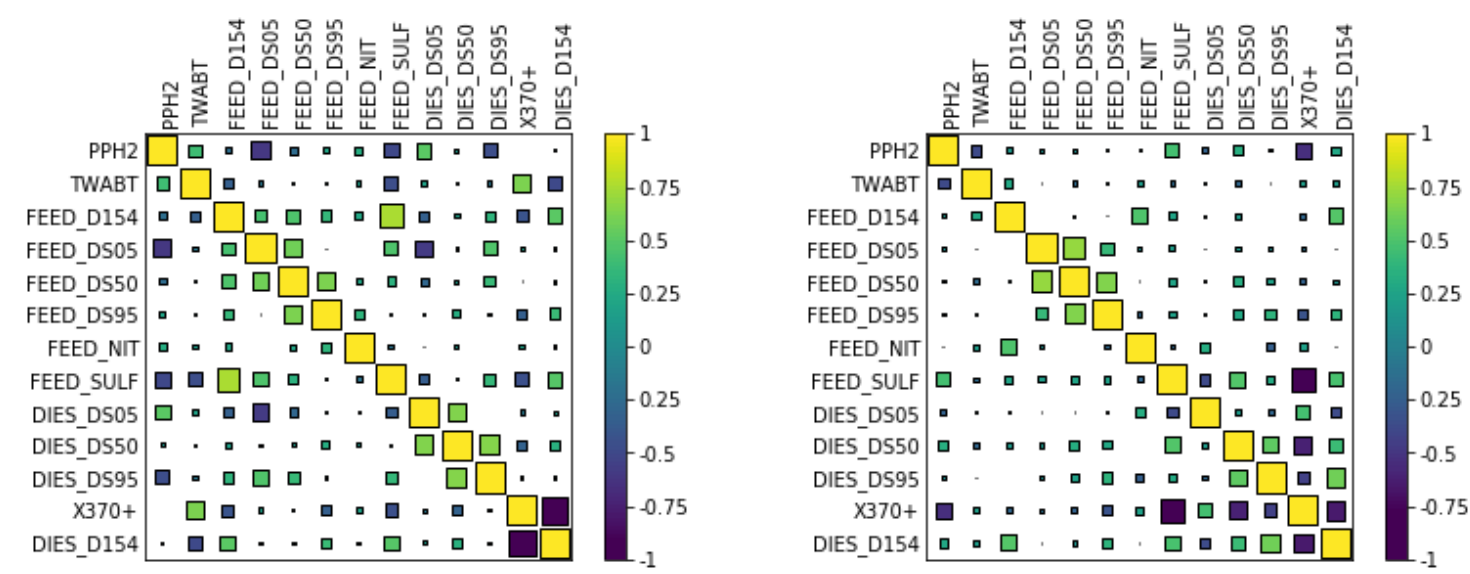

Fig. 1. Correlation matrix for two different catalysts. Left is the "old" catalyst $(3,177$ observations) and right is the "new" catalyst (1,004 observations).

(left), the largest correlation is with conversion (X370+), while the others are at least twice lower. For the new catalyst (right), the correlations are more homogeneous and new correlations appear as with DIES_DSxx. It is interesting to note that the correlation between input features can be high. For example, feedstock density (FEED_D154) is related to the distillation parameters (FEED_DSxx). Feed sulfur content and feed density are both a consequence of the origin of the crude oil. Similarly, a higher feed density will probably result in a higher cracked diesel density. It is important to note at this point that although correlated, those parameters do not carry the same information. It is also worth noticing that correlation between features depends on the data set. For example, FEED_D154 is correlated with FEED_SULF for the first catalyst but not the second. The same can be said for FEED_DS05 and PPH2. This can be explained by both the unrepresentative data sampling on a small number of plants, and by a different performance of the catalyst that requires different tuning of the hydrocracking unit, and thus mathematically changes the relationship between feed, products properties and process parameters.

\subsection{Content of the paper}

In the next section, we start by building a good predictive model for the source data set (Section 2). Different models are tested and two are chosen to be transferred: linear model for its simplicity and kriging for its good predictive results. For transferring the linear regression model, two methods are used. The first one is a parametric approach (Section 3.1.1) directly inspired from Bouveyron and Jacques (2010). The second approach is Bayesian (Section 3.1.2) and consists in using the source parameter as prior 
information for the target model. For the kriging model, a Bayesian transfer learning approach is proposed in Section 3.2, which is the main methodological contribution of the paper. This last method offers excellent results and allows the number of target observations required to be greatly reduced. Section 4 concludes the paper with some perspectives.

\section{Regression models for the source data}

The goal of this paper is to propose transfer learning methods for transferring regression models from an old catalyst to a new one. Obviously, the transfer method depends on the type of regression model. The objective in this Section is to find the best performing model type for our data without thinking about the transfer that will follow. Consequently, several regression models have been tested on the source data: Linear Model (LM, Seber and Lee (2012)), Random Forest (RF, Breiman (2001)), Gradient Boosting (GB, Friedman (2001)), Multi-layer Perceptron (MLP, Rumelhart, Hinton, and R. J. Williams (1985)), Support Vector Regression (SVR, Drucker et al. (1996)) and kriging (Cressie (1990)). Only two of them will be considered for transfer learning. Kriging is the model we will mainly focus on, thus we will present it before detailing the results and retained models.

\subsection{Kriging}

The kriging model, also known as Gaussian Process regression (Rasmussen and C. K. Williams (2006)), can be written as follows:

$$
y_{i}=m\left(\boldsymbol{x}_{i}\right)+Z\left(\boldsymbol{x}_{i}\right)
$$

where $m\left(\boldsymbol{x}_{i}\right)$ is the trend of the model and $Z\left(\boldsymbol{x}_{i}\right)$ is a second-order Gaussian stationary process such that:

$$
\operatorname{cov}\left(Z\left(\boldsymbol{x}_{i}\right), Z\left(\boldsymbol{x}_{i^{\prime}}\right)\right)=C\left(\left|\boldsymbol{x}_{i}-\boldsymbol{x}_{i^{\prime}}\right|\right) .
$$

Its covariance depends only on the distance between the observations $\boldsymbol{x}_{i}$ and $\boldsymbol{x}_{i^{\prime}}$.

In this work, the shape of the trend is assumed to be known, i.e. $m\left(\boldsymbol{x}_{i}\right)=\sum_{j=0}^{d} \beta_{j} f_{j}\left(\boldsymbol{x}_{i}\right)$, where the functions $f_{j}$ are known, and only $\beta_{j}$ should be estimated. The $f_{j}$ are chosen to be the identity function for $j>0, f_{0}\left(\boldsymbol{x}_{i}\right)=1$ and $d=p$ the number of features, which is a common choice corresponding to a linear trend. Let remark that in the field Gaussian Process regression, the trend is often assumed to be null, $m\left(\boldsymbol{x}_{i}\right)=0$. This model with null trend will be referred to as GP in the following, whereas the term kriging will be use for a GP with a linear trend. It will be seen that using a trend improves the predictive results for the modeling of the hydrocracking process. Moreover, kriging model allows to have explicit information about the phenomenon, what is useful for the process engineers, and to have a better quality of extrapolation.

The shape of the covariance function is also assumed to be known:

$$
\begin{aligned}
C\left(h_{1}, \ldots, h_{p}\right) & =\sigma^{2} R_{\boldsymbol{\theta}, \tau}\left(h_{1}, \ldots, h_{p}\right)=\sigma^{2} \prod_{j=1}^{p} g\left(h_{j}, \theta_{j}\right)+\delta_{\mathbf{0}}\left(h_{1}, \ldots, h_{p}\right) \tau \\
\delta_{\mathbf{0}}\left(h_{1}, \ldots, h_{p}\right) & =\left\{\begin{array}{l}
1 \text { if }\left(h_{1}, \ldots, h_{p}\right)=(0, \ldots, 0), \\
0 \text { else }
\end{array}\right.
\end{aligned}
$$


lapteff, L. et al.

with $\sigma^{2}$ the variance of the process, $\tau$ is a constant introduced to treat discontinuity known as the nugget effect (Cressie (1988)), and $\boldsymbol{\theta}=\left(\theta_{j}\right)_{j=1, \ldots, p}$ the parameters of the covariance function $g$, which is chosen to be the Matern $5 / 2$ covariance function: $g\left(h_{j}, \theta_{j}\right)=\left(1+\sqrt{5}\left|h_{j}\right| / \theta_{j}+5 h_{j}^{2} /\left(3 \theta_{j}^{2}\right)\right) \exp \left(-\sqrt{5}\left|h_{j}\right| / \theta_{j}\right)($ Stein $(2012))$. These are classic choices (Roustant, D. Ginsbourger, and Deville (2012)).

Parameter estimation is traditionally done by maximum likelihood, and prediction for a new observation $\boldsymbol{x}_{0}$ with the kriging model is achieved by looking for the best linear unbiased predictor $\widehat{y}_{0}$. It is obtained by finding $\boldsymbol{\lambda}\left(\boldsymbol{x}_{0}\right)=\left(\lambda_{1}\left(\boldsymbol{x}_{0}\right), \ldots, \lambda_{n}\left(\boldsymbol{x}_{0}\right)\right)^{T}$ minimizing $\mathbf{E}\left[\left(Z\left(\boldsymbol{x}_{0}\right)-\sum_{i=1}^{n} \lambda_{i}\left(\boldsymbol{x}_{0}\right) Z\left(\boldsymbol{x}_{i}\right)\right)^{2}\right]$. The solution is given by $\widehat{\boldsymbol{\lambda}}\left(\boldsymbol{x}_{0}\right)=\boldsymbol{C}^{-1} c\left(\boldsymbol{x}_{0}\right)$ with $\boldsymbol{C}=\left(C\left(\left|\boldsymbol{x}_{i}-\boldsymbol{x}_{i^{\prime}}\right|\right)\right)_{1 \leq i, i^{\prime} \leq n}$ and $c\left(\boldsymbol{x}_{0}\right)=\left(C\left(\left|\boldsymbol{x}_{0}-\boldsymbol{x}_{i}\right|\right)\right)_{1 \leq i \leq n}$.

Finally, the prediction $\widehat{y}_{0}$ is:

$$
\begin{aligned}
\widehat{\boldsymbol{\beta}} & =\left(f(\boldsymbol{X})^{T} \boldsymbol{C}^{-1} f(\boldsymbol{X})\right)^{-1} f(\boldsymbol{X})^{T} \boldsymbol{C}^{-1} \boldsymbol{y}, \\
\widehat{y}_{0} & =f\left(\boldsymbol{x}_{\mathbf{0}}\right) \widehat{\boldsymbol{\beta}}+c\left(\boldsymbol{x}_{\mathbf{0}}\right)^{T} \boldsymbol{C}^{-1}(\boldsymbol{y}-f(\boldsymbol{X}) \widehat{\boldsymbol{\beta}}),
\end{aligned}
$$

where $\boldsymbol{X}$ is the matrix whose $n$ rows are the $\boldsymbol{x}_{i}, \boldsymbol{y}=\left(y_{i}\right)_{i=1, \ldots, n}$ and $\widehat{\boldsymbol{\beta}}$ estimation of $\boldsymbol{\beta}$.

A detailed description of the Kriging model is presented in Roustant, D. Ginsbourger, and Deville (2012).

\subsection{Experimental settings}

In order to reduce the impact of outliers, the source data are normalized according to the median for centering and the interquartile range for reduction. To evaluate model quality, the source data set is split into a training and test set according to the Kennard and Stone algorithm (Kennard and L. Stone (1969)). This is an iterative algorithm. It is initialized with a training data set composed of the two most distant points of the complete data set and a test set composed of the remaining points. Then, at each iteration one point $\boldsymbol{x}_{i}$ is moved from the test data set to the training data set such as $\boldsymbol{x}_{i}=\underset{\boldsymbol{x}_{i} \in \text { Test }}{\operatorname{argmax}}\left(\underset{\boldsymbol{x}_{i^{\prime}} \in \text { Training }}{\min } \operatorname{dist}\left(\boldsymbol{x}_{i}, \boldsymbol{x}_{i^{\prime}}\right)\right)$ until the training data set reaches a pre-defined size. This ensures a training set with the maximum amount of information. The sizes of the test and train sets are both $50 \%$ of the complete data set size. The training and test sets are no longer normalized, and a second normalization is performed on the training set. Parameters of this normalization are then used to normalize the test set. For the Section 3, the target data set is also normalized according to these parameters. To complete preprocessing, an outlier detection is achieved on training set using the Local Outlier Factor method (Breunig et al. (2000)), with 10 nearest neighbors. The threshold is chosen such that the observations with a distant LOF score compared to the other are ranked as outlier. This leads to a threshold of 1.5 and about $2.5 \%$ of the observations are classified as outliers.

RF, GB, SVR and MLP have hyperparameters to tune: number of trees and their depths for RF and GB, number and size of the layers and activation function for MLP, size of the epsilon-tube and regularization parameter for SVR. A grid search for these hyperparameters is used with a range specified in Table 2. For each model, the hyperparameter combination that minimizes the RMSE score $\left(\sqrt{\frac{1}{n} \sum_{i=1}^{n}\left(\hat{y}_{i}-y_{i}\right)^{2}}\right.$ where $\hat{y}_{i}$ is the predicted value) evaluated with 10 fold cross-validation is selected. For all these 
Table 2. Hyperparameters and their values tested by grid search for the different models.

\begin{tabular}{lcc}
\hline Model & Hyperparameter & Value \\
\hline \multirow{2}{*}{ RF } & $n_{\text {tree }}$ & $\{10 k\}_{k=1, \ldots, 10}$ \\
& $\max _{\text {depth }}$ & $\{5 k\}_{k=1, \ldots, 6}$ \\
\hline \multirow{2}{*}{ GB } & $n_{\text {tree }}$ & $\{10 k\}_{k=1, \ldots, 10}$ \\
& max $_{\text {depth }}$ & $\{5 k\}_{k=1, \ldots, 6}$ \\
\hline \multirow{2}{*}{ MLP } & $n_{\text {layers }}$ & $\{1,2,3\}$ \\
& size $_{\text {layer }}$ & $\{5,8,12,16\}$ \\
& $f_{\text {activ }}$ & $\{$ relu, tanh $\}$ \\
\hline \multirow{2}{*}{ SVR } & $\epsilon$ & $\left\{10^{-4}, 10^{-3}, 10^{-2}\right\}$ \\
& $\mathrm{C}$ & $\{1,5,10,15,20,25,30\}$ \\
\hline
\end{tabular}

models (RF, GB, SVR and MLP), the scikit-learn implementation is used (Pedregosa et al. (2011)).

For the kriging model and GP, the DiceKriging package from R (Roustant, D. Ginsbourger, and Deville (2012)) is used to fit the model. This package uses the BFGS algorithm to maximize the likelihood. The chosen hyperparameters are mentioned in Section 2.1. As a nugget effect is used, it has to be estimated. Universal kriging is considered, it means that the trend parameters are unknown and are estimated. The upper bound of the $\boldsymbol{\theta}$ parameters is set to twice the difference between the maximum and minimum values for each feature, as suggested in Roustant, D. Ginsbourger, and Deville (2012).

\subsection{Model comparison}

The models are evaluated on the test set according to three criteria: 1) the RMSE score, 2 ) the percentage of observations for which the prediction error $\left(\left|y_{i}-\hat{y}_{i}\right|\right)$ is less than $0.005,3)$ the percentage of observations for which the prediction error is less than 0.0025 . The limit of 0.005 on the prediction error is commonly used in the field of oil product density prediction, knowing that experimental measurement accuracy of DIES_D154 is 10 times smaller (0.0005).

The results are given in Figure 2 and Table 3. Globally, the results are quite similar for all the models. For the three studied scores, the kriging model offers the best results with a RMSE score below 0.00229. With the no trend model (GP), the results are a little worse with an RMSE score of 0.00236 . The RF, MLP, SVR and GB models offer slightly poorer results and are all similar with RMSE scores between 0.0024 and 0.0026 . The percentage of observations with an error lower than 0.005 is higher than $95 \%$ for these 6 models, which is quite satisfactory indeed. Although satisfactory, the results of LM are worse than those of the other models and have a bias by plant. However, it has the advantage of being an easily interpretable and understandable model, allowing the effects of each variable to be analyzed. Let finally notice that the prediction qualities per plant are nearly equivalent. This can be seen in Figure 2 where the colored dots represent the plants, named from A to $\mathrm{H}$.

Since the kriging model provides the best performance, it is chosen as a candidate to be transferred to a new catalyst. The method presented in Section 1.2 for transferring some of other models could be effective to answer the problem, but we focus on the 

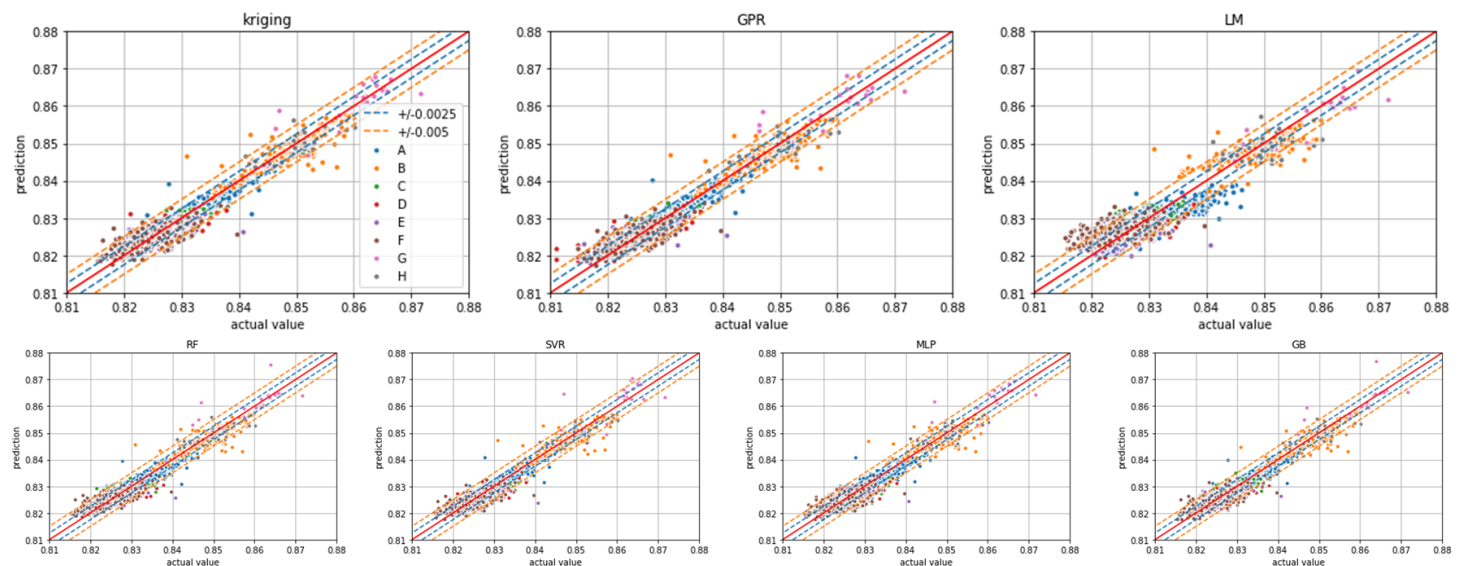

Fig. 2. Results of the different models fitted on the training source data, applied to test source data. The colored dots represent different plants.

Table 3. Scores of different models fitted on the training source data, applied to the test source data.

\begin{tabular}{lccccccc}
\hline Model & LM & RF & GB & MLP & SVR & kriging & GP \\
\hline RMSE & 0.00331 & 0.00248 & 0.00243 & 0.00259 & 0.00244 & 0.00229 & 0.00236 \\
$+/-0.0025$ & $59.9 \%$ & $76.5 \%$ & $76.8 \%$ & $73.0 \%$ & $78.6 \%$ & $80.5 \%$ & $79.0 \%$ \\
$+/-0.005$ & $87.3 \%$ & $95.1 \%$ & $95.5 \%$ & $95.0 \%$ & $95.5 \%$ & $96.2 \%$ & $95.9 \%$ \\
\hline
\end{tabular}

kriging model which offers the best performance on our data.

As the GP is the same model as the kriging but without trend, its transfer will also be considered. Additionally, we also select the linear model for its simplicity and interpretability. The next section describes transfer learning methods for the linear and kriging models.

\section{Transfer Learning for regression}

In this section, a Bayesian transfer learning method is proposed for the kriging model. For comparison, two transfer learning methods, Bayesian and parametric, are also considered for the linear model. The target data set on which the models have to be transferred is composed of 1,004 observations. Since, in practice, the goal is to be able to build a model with as few points as possible from the target catalyst, a subset of size $n_{t}$ is randomly extracted from the whole target data set. Different sizes of $n_{t}$ will be considered, and for each size, 10 randomly sampled data sets are considered and average results are presented. The quality of the model is evaluated with the RMSE score evaluated on an independent test set composed of 804 data points (different from the $n_{t}$ data used for transfer). In the present industrial context, a model is considered satisfactory if the RMSE score is lower than 0.005. For memory, the respective RMSE scores on the source data set for the linear and kriging models are 0.0033 and 0.0023 (Table 3), respectively, which is quite satisfactory.

Variables referring to the source will be indexed by "s" $\left(\boldsymbol{X}_{s}, \boldsymbol{y}_{s}, \ldots\right)$ while those 
referring to the target will be indexed by "t" $\left(\boldsymbol{X}_{t}, \boldsymbol{y}_{t}, \ldots\right)$.

\subsection{Transfer learning for the linear model}

The model for the source catalyst is

$$
y_{i}=\beta_{s 0}+\sum_{j=1}^{p} \beta_{s j} x_{i j}+\epsilon_{i}
$$

with $\epsilon_{i} \sim \mathcal{N}\left(0, \sigma_{s}^{2}\right)$ and $p=12$. The maximum likelihood estimator on the source data gives the results in Section 2.3. Our goal is to estimate the same model, but for the target catalyst

$$
y_{i}=\beta_{t 0}+\sum_{j=1}^{p} \beta_{t j} x_{i j}+\epsilon_{i}
$$

with $\epsilon_{i} \sim \mathcal{N}\left(0, \sigma_{t}^{2}\right)$ and for which the available training data set is of a smaller size $n_{t}$.

\subsubsection{A parametric approach}

The first transfer method for the linear model is a parametric approach inspired from Bouveyron and Jacques (2010), in which some regression parameters are kept unchanged for the target model $\left(\beta_{s j}=\beta_{t j}\right.$ for some $j$ ), considering the influence doesn't change between both models, and then learn only other parameters. The standard deviation parameter $\sigma_{t}$ is assumed to be equal to $\sigma_{s}$.

If $\mathcal{M}$ is the set of index of parameters to be modified, then $\beta_{t j}=\beta_{s j}$ for $j \in$ $\{1, \ldots, p\} \backslash \mathcal{M}$ and $\beta_{t j}=\lambda_{j} \beta_{s j}$ for $j \in \mathcal{M}$. Then, only a reduced number of parameters have to be estimated for the target model. The challenge with this approach is how to chose $\mathcal{M}$, in particular the number of parameters and which ones. In the first step, we will assume $\# \mathcal{M}$ is known and we will select the best parameters by leaveone-out cross validation (LOOCV) on the $n_{t}$ target points. When $\# \mathcal{M}=1$, the best parameter is the one minimizing the RMSE by LOOCV. For $\# \mathcal{M}>1$, we decided not to test all possible combinations and rather to identify successively the next best parameter to modify. The final model is obtained by choosing the values of the $\# \mathcal{M}$ parameters minimizing the RMSE by LOOCV.

The results are presented in the left panel of Figure 3 for $\# \mathcal{M} \in\{1,3,8,13\}$. With this approach, a performing model can be fitted with less points than if a totally new model is learned (Figure 3, left). For example, with 10 observations and modifying 1 or 3 parameters, RMSE is smaller than the objective of 0.005. In contrast, to achieve this result by learning the model from scratch (on all parameters), 30 observations are needed. Changing a small number of parameters, like one or two, is more efficient for small training sets but worse when a lot of data is available where a plateau is quickly reached. When $\# \mathcal{M}$ is higher, the number of points needed to get a satisfactory score is higher but the prediction accuracy keeps improving. Another remark is that for a given $\# \mathcal{M}$, the selected parameters change with changing sample size. In other words, we are not able to define the best parameters to transform with a few points. 
The next step would be to decide on the optimal size of $\mathcal{M}$. Our idea is to select, among all the values of $\# \mathcal{M}$, the one with the best RMSE (LOOCV) for each $n_{t}$. Thus, for each $n_{t}$, we compare the RMSE scores for the different values of $\# \mathcal{M}$ and we keep the value of $\# \mathcal{M}$ offering the best results. The value of $\# \mathcal{M}$ will potentially be different for each sample size. As shown in Figure 3 (right), this approach does not give the lowest RMSE on the test set and is only marginally better than the model learned from scratch. So far, we did not find an effective method for determining the parameters and the number of parameters to be modified. For this reason, Bayesian inference is explored.
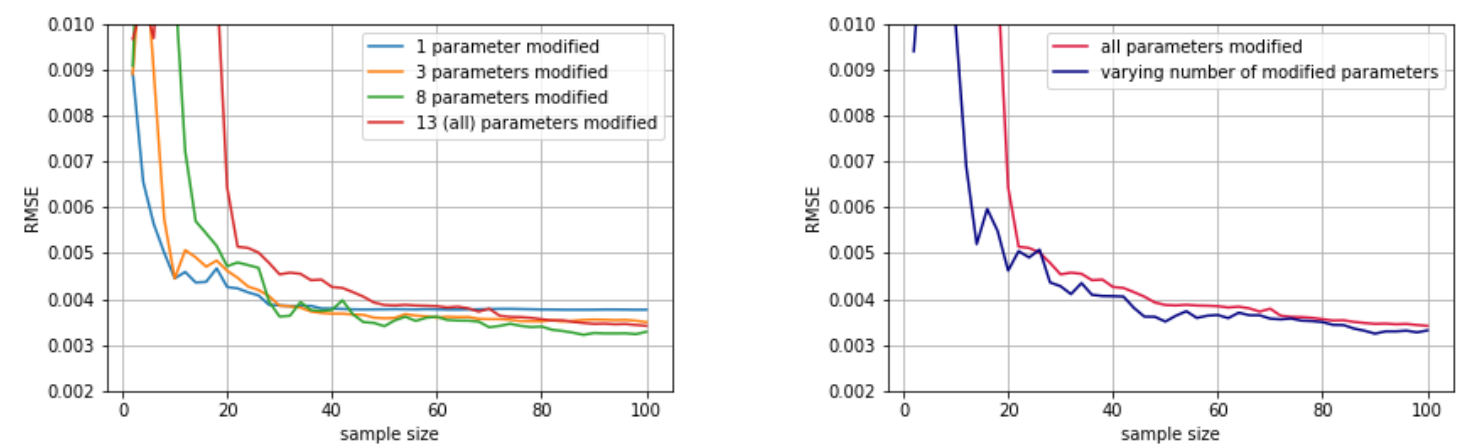

Fig. 3. Graphs showing the change of RMSE according to $n_{t}$. On the left, the parameters to modify were chosen by cross validation. On the right, cross validation is also used to determine the number of parameters to be modified. The $y$-axis has been cut for better readability, as very high scores do not interest us.

\subsubsection{A Bayesian approach}

In this section, a Bayesian approach is used to learn parameters for the target linear model. The linear model for the target is:

$$
\boldsymbol{y}=\boldsymbol{\beta}_{t} \boldsymbol{X}+\boldsymbol{\epsilon}
$$

where $\boldsymbol{\beta}_{t}=\left(\beta_{t 0}, \beta_{t 1}, \ldots, \beta_{t p}\right)^{T}$ is a random variable of prior density $\pi\left(\boldsymbol{\beta}_{t}\right), \boldsymbol{X}$ is the design matrix into which a first unity column has been added. The Bayes Theorem gives that the posterior of $\boldsymbol{\beta}_{t}$ is

$$
\pi\left(\boldsymbol{\beta}_{t} \mid \boldsymbol{y}_{t}, \boldsymbol{X}_{t}\right)=\frac{\pi\left(\boldsymbol{\beta}_{t}\right) f\left(\boldsymbol{y}_{t} \mid \boldsymbol{\beta}_{t}, \boldsymbol{X}_{t}\right)}{f\left(\boldsymbol{y}_{t} \mid \boldsymbol{X}_{t}\right)}
$$

where $\left(\boldsymbol{y}_{t}, \boldsymbol{X}_{t}\right)$ are the target observations.

Different prior distributions $\pi\left(\boldsymbol{\beta}_{t}\right)$ are considered. The first one is the well known Zellner's prior (Zellner (1986)), also known as g-prior, for the parameters $\boldsymbol{\beta}_{t}$ :

$$
\pi\left(\boldsymbol{\beta}_{t}\right)=\mathcal{N}\left(\widehat{\boldsymbol{\beta}}_{s}, g \sigma_{t}^{2}\left(\boldsymbol{X}_{t}^{T} \boldsymbol{X}_{t}\right)^{-1}\right),
$$


where $\widehat{\boldsymbol{\beta}}_{s}$ is the maximum likelihood estimator (MLE) learned on the source data. By using such a prior, only the mean of the prior distribution depends on the source data. The structure of the prior covariance of $\boldsymbol{\beta}_{t}$ depends on the target data, and a scalar parameter $g$ allows the impact of the prior distribution to be tuned. Notice that the posterior's mean using such a prior is a weighted average between the MLE for source data and the MLE for target data: $\widehat{\boldsymbol{\beta}_{t}}=\frac{1}{g+1}\left(g \widehat{\boldsymbol{\beta}}_{M L E, t}+\widehat{\boldsymbol{\beta}_{s}}\right)$.

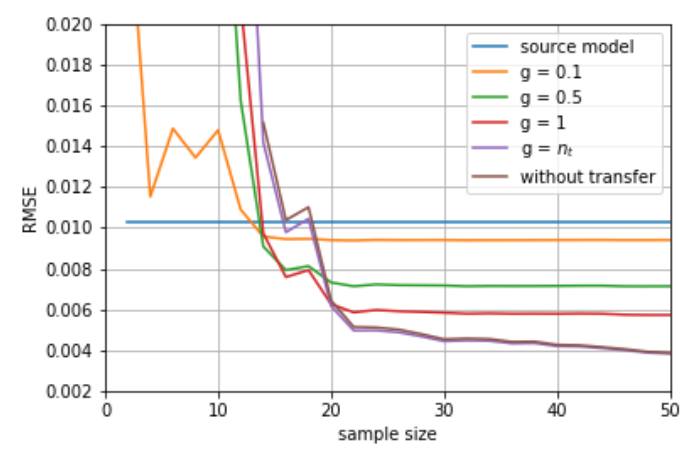

Fig. 4. Comparison between an estimation of $\boldsymbol{\beta}_{t}$ with a Bayesian approach and a g-prior for different values of $\mathrm{g}$, and a model learned without any prior.

The results are presented in Figure 4 . With this prior, the results are not satisfactory irrespective of the value of $g$. For a target sample size lower than 15, although the transferred linear models have a lower RMSE than the model estimated without transfer (directly from the target data), the source model remains better (blue line on Figure 4). For a larger sample size, the results are better for the transferred linear model, but the desired RMSE score of 0.005 is not reached.

With this Zellner's prior, the source data set acts only on the mean of the prior distribution. One idea to improve the results is to increase the information transferred by also acting on prior covariance. Indeed, when $n_{t}$ is small, the covariance structure cannot be well estimated. We consequently proposed the following prior:

$$
\pi\left(\boldsymbol{\beta}_{t}\right)=\mathcal{N}\left(\widehat{\boldsymbol{\beta}}_{s}, g \sigma_{s}^{2}\left(\boldsymbol{X}_{s}^{T} \boldsymbol{X}_{s}\right)^{-1}\right),
$$

in which the covariance structure is now estimated from the source data. The mean of the corresponding posterior distribution is equal to:

$$
\widehat{\boldsymbol{\beta}_{t}}=\left(\boldsymbol{X}_{t}^{T} \boldsymbol{X}_{t}+\sigma_{t}^{2} g^{-1} \boldsymbol{\Sigma}_{s}^{-1}\right)^{-1}\left(\boldsymbol{X}_{t}^{T} \boldsymbol{y}_{t}+\sigma_{t}^{2} g^{-1} \boldsymbol{\Sigma}_{s}^{-1} \widehat{\boldsymbol{\beta}}_{s}\right),
$$

with $\boldsymbol{\Sigma}_{s}=\sigma_{s}^{2}\left(\boldsymbol{X}_{s}^{T} \boldsymbol{X}_{s}\right)^{-1}$. We notice that when $g \rightarrow \infty$ the posterior mean tends to the MLE learned on target observation. When $g \rightarrow 0$ the posterior mean tends to the prior mean. The RMSE scores are computed for different values of $g$ in Figure 5 (left panel). With this prior, we pass under the threshold of 0.005 for the RMSE score for all $g$ values greater than 1. Furthermore, such $g$ values lead to better results than those obtained when estimated the model directly from the target data without transfer. However, $g$ value as to be chosen and we propose a pragmatic empirical strategy. 

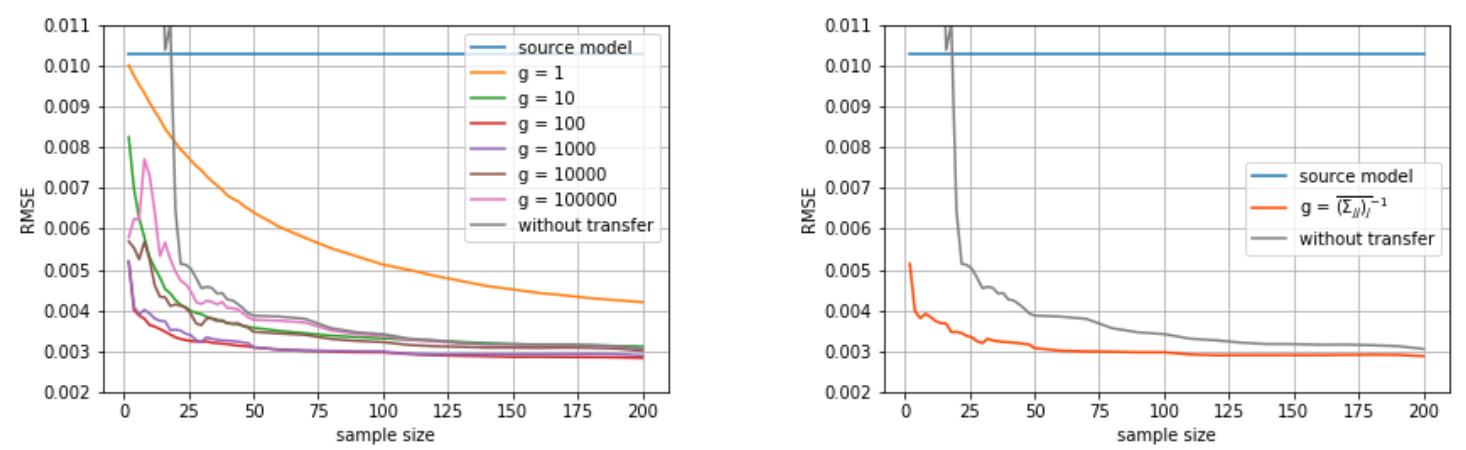

Fig. 5. Impact of $g$ on the Bayesian linear model. The graph shows the change in RMSE for different values of $g$ according to $n_{t}$.

Since data are normalized, elements of parameter $\boldsymbol{\beta}_{\boldsymbol{t}}$ take values close to $[-1,1]$. In order to allow a parameter to change in this range, the prior variance should be close to 1 . A suitable $g$ value should be around of the inverse of the average of the diagonal elements of $\boldsymbol{\Sigma}_{s}$. Following this strategy leads to a $g$ value approximately equal to 800 in our experiment. The corresponding results are shown in the right panel of Figure 5.

With this approach, the RMSE scores are always lower than those obtained without transfer. Moreover, only 5 target points are needed to reach the industrial constraint of a RMSE lower than 0.005. In comparison, estimating the target model without transfer learning will need at least 50 observations.

\subsection{Transfer learning for the kriging Model}

Following these encouraging results in the Bayesian transfer of linear model (only 5 points are needed for the target data), we will now focus on transferring the kriging model using a Bayesian approach. The kriging model (2) is composed of two parts, a trend part and a Gaussian Process part:

$$
y_{i}=\Sigma_{j=0}^{d} \beta_{j} x_{i j}+Z\left(\boldsymbol{x}_{i}\right) .
$$

Inspired from the Bayesian transfer for the linear model, a first Bayesian kriging model is considered with a prior on the trend part only (Section 3.2.1). In a second step, we additionally consider a prior distribution for the covariance of the Gaussian process (Section 3.2.2).

\subsubsection{A Bayesian transfer on trend parameters only}

Here, the covariance function $C$ is assumed to be known and identical to the covariance function of the source model. Consequently, its parameters $\boldsymbol{\theta}_{t}, \sigma_{t}$ and $\tau_{t}$ are equal to $\hat{\boldsymbol{\theta}}_{s}, \hat{\sigma}_{s}$ and $\hat{\tau}_{s}$, respectively. The covariance of the Gaussian Process used for prediction is calculated only for observations from the target data set:

$\boldsymbol{C}_{t}=\left(C\left(\left|\boldsymbol{x}_{t i}-\boldsymbol{x}_{t i^{\prime}}\right|\right)\right)_{1 \leq i, i^{\prime} \leq n_{t}}$. 
As in the Bayesian transfer of the linear model, a Gaussian prior distribution is considered for $\boldsymbol{\beta}_{t}$, with mean $\widehat{\boldsymbol{\beta}}_{s}$ and variance $\boldsymbol{\Sigma}_{s}$ modified by a factor $g$ :

$$
\pi\left(\boldsymbol{\beta}_{t}\right)=\mathcal{N}\left(\widehat{\boldsymbol{\beta}}_{s}, g \boldsymbol{\Sigma}_{s}\right),
$$

where $\boldsymbol{\Sigma}_{s}=\left(\boldsymbol{X}_{s}^{T} \boldsymbol{C}_{s}^{-1} \boldsymbol{X}_{s}\right)^{-1}, \boldsymbol{C}_{s}=\left(\widehat{\sigma}_{s}^{2} R_{\widehat{\boldsymbol{\theta}}_{s}, \widehat{\tau}_{s}}\left(\left|\boldsymbol{x}_{s i}-\boldsymbol{x}_{s i^{\prime}}\right|\right)\right)_{1 \leq i, i^{\prime} \leq n_{s}}$.

This prior leads to the following posterior distribution for $\boldsymbol{\beta}_{t}$ (as used in Helbert, Dupuy, and Carraro (2009)):

$$
\begin{gathered}
\pi\left(\boldsymbol{\beta}_{t} \mid \boldsymbol{y}, \boldsymbol{X}\right)=\mathcal{N}\left(\widehat{\boldsymbol{\beta}}_{s}+g \boldsymbol{\Sigma}_{s} \boldsymbol{X}^{T}\left(g \boldsymbol{X} \boldsymbol{\Sigma}_{s} \boldsymbol{X}^{T}+\boldsymbol{C}_{s}\right)^{-1}\left(\boldsymbol{y}-\boldsymbol{X} \widehat{\boldsymbol{\beta}}_{s}\right),\right. \\
\left.g \boldsymbol{\Sigma}_{s}-g \boldsymbol{\Sigma}_{s} \boldsymbol{X}^{T}\left(g \boldsymbol{X} \boldsymbol{\Sigma}_{s} \boldsymbol{X}^{T}+\boldsymbol{C}_{s}\right)^{-1} g \boldsymbol{X} \boldsymbol{\Sigma}_{s}\right)
\end{gathered}
$$

Figure 6 presents the corresponding RMSE scores, for different values of $g$. Irrespective of the value of $g$, the transferred kriging models outperform the kriging model estimated on the target data only.

Similar to the linear model, $g$ is chosen to be equal to the inverse of the average of the diagonal of $\boldsymbol{\Sigma}_{s}(g \simeq 200)$. We notice that other choices for $g$ could lead to even better results, but the choice of $g$ is difficult when using a small target sample, and we advise on considering our heuristic value for $g$ rather than trying to tune it by cross-validation.

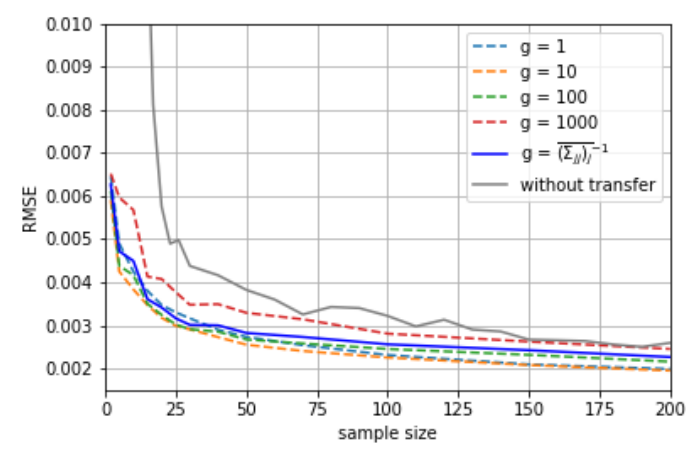

Fig. 6. Impact of $g$ on the Bayesian kriging model. The graph shows the change in RMSE for different values of $g$ according to $n_{t}$.

With Bayesian transfer on the trend, 5 target observations are enough to reach a RMSE score of 0.005 instead of about 25 for a kriging model learned on the target data only. The best RMSE score reached with the transferred linear model with 50 points $(0.003$, Figure 5$)$, is obtained with only 25 points only and is outperformed when increasing the number of points. In addition to reducing the number of points required to learn a model that meets the performance criteria, the Bayesian transfer of the trend of a kriging model also improves the performance of the model for a larger number of points.

3.2.2. A fully Bayesian approach

In this section, we add a prior distribution on the covariance function parameters $\boldsymbol{\theta}_{t}, \sigma_{t}$ and $\tau_{t}$. 
Prior distribution The choice of prior for $\boldsymbol{\beta}_{t}$ is still a Gaussian distribution, and the prior for the other parameters are Gamma distributions. The Gamma distributions are such that the mean and variance are those of the source parameters. The mean and variance of a random variable following a Gamma distribution $\mathcal{G}(a, b)$ of parameter of shape a and parameter of scale b are respectively $a b$ and $a b^{2}$. This leads to the following prior:

$$
\begin{aligned}
\pi\left(\boldsymbol{\beta}_{t}, \boldsymbol{\theta}_{t}, \sigma_{t}, \tau_{t}\right) & =\pi\left(\boldsymbol{\beta}_{t} \mid \boldsymbol{\theta}_{t}, \sigma_{t}, \tau_{t}\right) \pi\left(\boldsymbol{\theta}_{t}, \sigma_{t}, \tau_{t}\right), \\
\pi\left(\boldsymbol{\beta}_{t} \mid \boldsymbol{\theta}_{t}, \sigma_{t}, \tau_{t}\right) & =\mathcal{N}\left(\widehat{\boldsymbol{\beta}}_{s}, g \boldsymbol{\Sigma}_{s}\right), \\
\pi\left(\boldsymbol{\theta}_{t}, \sigma_{t}, \tau_{t}\right) & =\prod_{j} \pi\left(\theta_{t j}\right) \times \pi\left(\sigma_{t}\right) \times \pi\left(\tau_{t}\right), \\
\pi\left(\theta_{t j}\right) & =\mathcal{G}\left(\frac{\hat{\theta}_{s j}^{2}}{\operatorname{Var}\left(\hat{\theta}_{s j}\right)}, \frac{\operatorname{Var}\left(\hat{\theta}_{s j}\right)}{\hat{\theta}_{s j}}\right), \\
\pi\left(\sigma_{t}\right) & =\mathcal{G}\left(\frac{\hat{\sigma}_{s}^{2}}{\operatorname{Var}\left(\hat{\sigma}_{s}\right)}, \frac{\operatorname{Var}\left(\hat{\sigma}_{s}\right)}{\hat{\sigma}_{s}}\right), \\
\pi\left(\tau_{t}\right) & =\mathcal{G}\left(\frac{\hat{\tau}_{s}^{2}}{\operatorname{Var}\left(\hat{\tau}_{s}\right)}, \frac{\operatorname{Var}\left(\hat{\tau}_{s}\right)}{\hat{\tau}_{s}}\right) .
\end{aligned}
$$

With these priors, no closed form exists for the posterior distribution and a MCMC algorithm is used to estimate the posterior distributions. Parameter $g$ and matrix $\boldsymbol{\Sigma}_{s}$ are chosen in the same manner as in the previous section.

Markov Chain Monte Carlo (MCMC) algorithm The goal is to approximate the posterior distribution of $\left(\boldsymbol{\beta}_{t}, \boldsymbol{\theta}_{t}, \sigma_{t}, \tau_{t}\right)$. The MCMC algorithm is an iterative algorithm that generates a Markov chain whose stationary distribution is the desired posterior. At each iteration, new values for the parameters are generated and we note the values of parameters $\boldsymbol{\beta}_{t}^{(q)}, \boldsymbol{\theta}_{t}^{(q)}, \sigma_{t}^{(q)}, \tau_{t}^{(q)}$ at iteration $(q)$. The MCMC algorithm used is a Metropolis Hastings within Gibbs algorithm (Tierney (1994)), which is detailed below.

At each iteration, the parameters are updated sequentially. For any scalar parameter $\rho$, a new value $\rho^{\text {new }}$ is proposed such that $\rho^{\text {new }}=\rho^{(q)}+r$ where $r$ is randomly drawn according to a centered Gaussian distribution of variance specific to each parameter. The idea of Metropolis-Hastings is to accept $\rho^{\text {new }}$ as the new value if its posterior is better than that of $\rho^{(q)}$, and randomly otherwise. We compute the ratios of the posterior as the ratio of the product between the likelihood and the prior, with all other parameters remaining unchanged and $\rho^{(q+1)}$ chosen to be $\rho^{\text {new }}$ with probability $\min (1$, ratio $)$ and $\rho^{(q)}$ otherwise. The variance of $r$ is chosen empirically so that the acceptance rates are between $20 \%$ and $60 \%$ for each parameter during all the iterations. Consequently, there is a need to run the algorithm a few times in order to tune these standard deviations. In our application, the following standard deviation was considered: $\xi_{\beta_{t j}}=0.2, \xi_{\theta_{t j}}=1$ and $\xi_{\sigma_{t}}=\xi_{\tau_{t}}=0.03$.

The inputs of the algorithm are the target data $\boldsymbol{X}_{t}, \boldsymbol{y}_{t}$ and the prior distribution $\pi\left(\boldsymbol{\beta}_{t}, \boldsymbol{\theta}_{t}, \sigma_{t}, \tau_{t}\right)$ defined above. Let $\mathcal{N}(\cdot ; \boldsymbol{\mu}, \boldsymbol{\Sigma})$ be the multivariate Gaussian probability density function (p.d.f) of parameter $(\boldsymbol{\mu}, \boldsymbol{\Sigma})$. Let $\mathcal{G}^{\left(\theta_{t j}\right)}(\cdot), \mathcal{G}^{\left(\sigma_{t}\right)}(\cdot), \mathcal{G}^{\left(\tau_{t}\right)}(\cdot)$ be the Gamma 
p.d.f. used as prior $\pi\left(\theta_{t j}\right), \pi\left(\sigma_{t}\right), \pi\left(\tau_{t}\right)$ for $j \in 1, \ldots, p$. Let $\boldsymbol{\rho}^{(q)}$ be $\left(\theta_{t 1}^{(q)}, \ldots, \theta_{t p}^{(q)}, \sigma_{t}^{(q)}, \tau_{t}^{(q)}\right)$ and $C\left(h_{1}, \ldots, h_{p} ; \boldsymbol{\rho}^{(q)}\right)=\sigma_{t}^{(q) 2} R_{\boldsymbol{\theta}_{t}^{(q)}, \tau_{t}^{(q)}}\left(h_{1}, \ldots, h_{p}\right)$.

Initialization of parameters, $\left(\boldsymbol{\beta}_{t}^{(0)}, \boldsymbol{\theta}_{t}^{(0)}, \sigma_{t}^{(0)}, \tau_{t}^{(0)}\right)$, is performed using the mean of the prior distribution. The algorithm for updating the parameters is slightly different for $\boldsymbol{\beta}_{t}$ that follows a Gaussian distribution and $\left\{\theta_{t j}\right\}_{j}, \sigma_{t}$ and $\tau_{t}$ that follow a Gamma distribution. The update of $\boldsymbol{\beta}_{t}^{(q)}$ is detailed in Algorithm 1. The update of $\left\{\theta_{t j}\right\}_{j}, \sigma_{t}$ and $\tau_{t}$ is detailed in Algorithm 2. The main difference is that, for the $\boldsymbol{\beta}_{t}$ case, the covariance of the Gaussian Process is not a function of the parameters and can be computed outside of the $\boldsymbol{\beta}_{t}$ update loop as $\left.\boldsymbol{C}=\sigma_{t}^{(q) 2} R_{\boldsymbol{\theta}_{t}^{(q)}, \tau_{t}^{(q)}}\left(\left|\boldsymbol{x}_{t i}-\boldsymbol{x}_{t i^{\prime}}\right|\right)\right)_{1 \leq i, i^{\prime} \leq n_{t}}$ with the values of the previous iteration $\boldsymbol{\theta}_{t}^{(q)}, \sigma_{t}^{(q)}, \tau_{t}^{(q)}$.
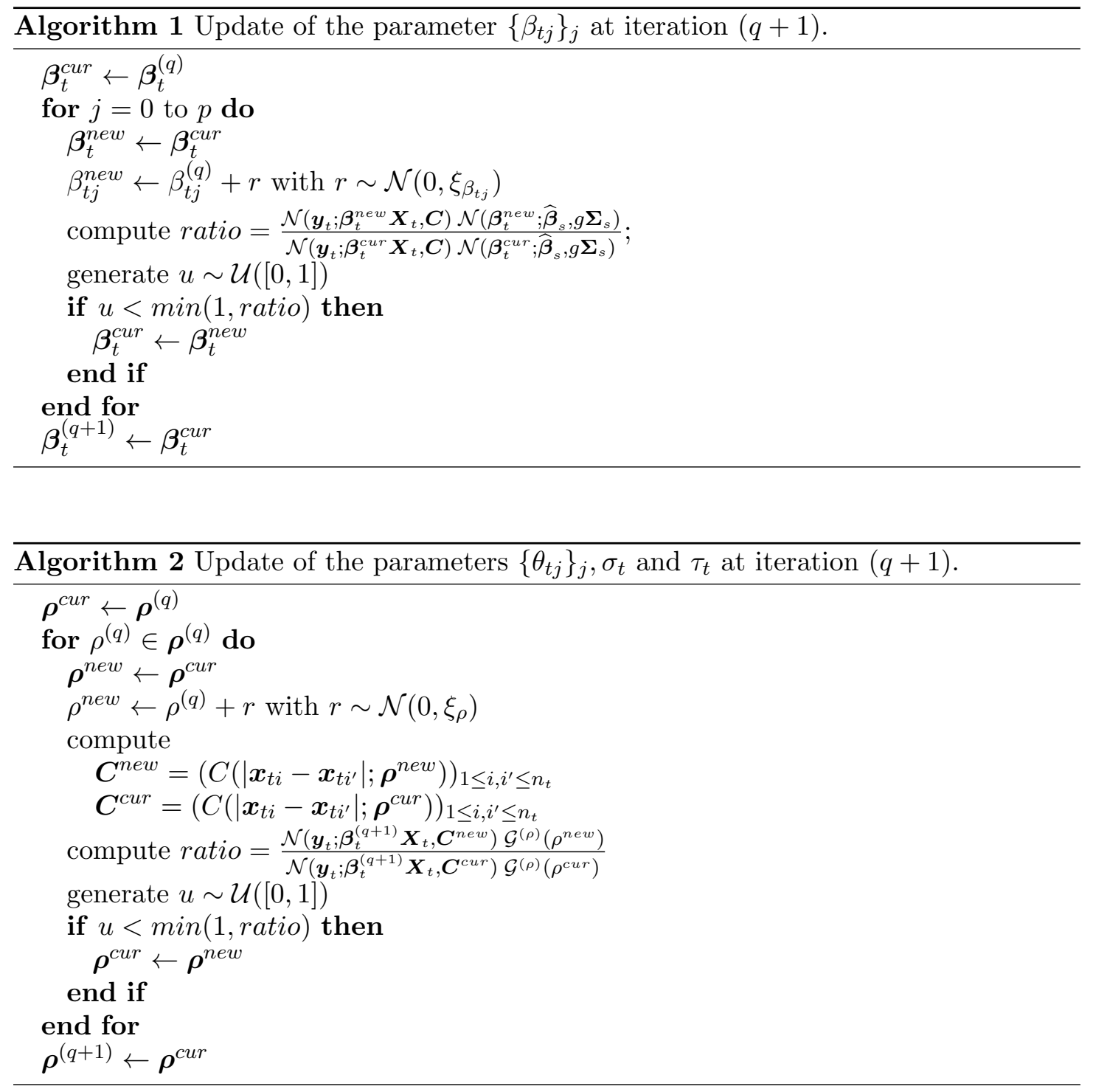
The number of iterations of the MCMC algorithm must be large enough so that the Markov chain converges to its stationary distribution. In practice, this number is fixed by observing the evolution of the Markov chain over the iterations and must have reached its stationary distribution for thousands of observations. Since the generated Markov chain needs a given time to reach its stationary distribution, the first iterations are dropped. The size of this burn-in period depends on how far $\left(\boldsymbol{\beta}_{t}^{(0)}, \boldsymbol{\theta}_{t}^{(0)}, \sigma_{t}^{(0)}, \tau_{t}^{(0)}\right)$ is from the mean of the posterior distribution. It is also fixed by observing the evolution of the Markov chain. In our application, 5,000 MCMC iterations with a burn-in period of 500 is considered. Finally, estimation of $\left(\widehat{\boldsymbol{\beta}}_{t}, \widehat{\boldsymbol{\theta}}_{t}, \widehat{\sigma}_{t}, \widehat{\tau}_{t}\right)$ is obtained by computing the empirical mean of the marginal posterior distribution.

Transfer Gaussian Process regression In the particular case of a kriging model with null trend, i.e. the classical Gaussian Process regression framework, some transfer techniques exists. B. Cao et al. (2010) propose an approach based on the following assumption for the covariance function:

$$
C^{\prime}\left(\left|\boldsymbol{x}_{i}-\boldsymbol{x}_{i^{\prime}}\right|\right)=\left\{\begin{array}{cl}
C\left(\left|\boldsymbol{x}_{i}-\boldsymbol{x}_{i^{\prime}}\right|\right) & \text { if }\left(\boldsymbol{x}_{i}, \boldsymbol{x}_{i^{\prime}}\right) \in X_{s}^{2} \text { or }\left(\boldsymbol{x}_{i}, \boldsymbol{x}_{i^{\prime}}\right) \in X_{t}^{2} \\
\lambda C\left(\left|\boldsymbol{x}_{i}-\boldsymbol{x}_{i^{\prime}}\right|\right) & \text { else }
\end{array}\right.
$$

With such an approach, the data from the source data set are not only used to fit the parameters but also for the prediction. This method is noted TGP hereafter. In comparison, the Bayesian approach we proposed, which is also applicable for GP since it is a particular kriging model, use for prediction only the target data. When applying our Bayesian transfer approach to transfer the GP, since there is no trend, only the parameters $\boldsymbol{\theta}$ must be estimated using the MCMC algorithm. In the sequel, both transfer methods (TGP and the Bayesian one) are compared for the Gaussian Process regression with zero mean.

Experimental results The results for kriging model with trend are presented in Figure 7. For a reduced number of target observations, and up to about 50 points, adding a prior on the covariance function parameter does not improve the results. But when the number of points is greater than 50, it slightly improves the results compared to the Bayesian kriging on trend parameters. This allows us to obtain particularly good predictions.

The results for the GP model (kriging without trend) are presented in Figure 8. First of all, in this application, without using transfer learning the GP is slightly better than the kriging model. Secondly, and as expected, the transferred GP model is more efficient than its non-transferred version. Finally, concerning the two approaches to achieve the transfer, Bayesian or TGP, they offer quite similar results.

In this application, the transferred GP and the kriging model lead to quite similar results. Consequently, we are tempted to conclude that the non null trend is not useful. However, from an industrial point of view, one of the objective is to build a model that can be extrapolated on a range of feature values relatively different from those observed in the training data set. In that sense, considering a model with a trend is generally more efficient (David Ginsbourger et al. (2009)) and kriging is preferred to GP without trend. Moreover, the trend contains explicit information on the phenomenon, which is an 


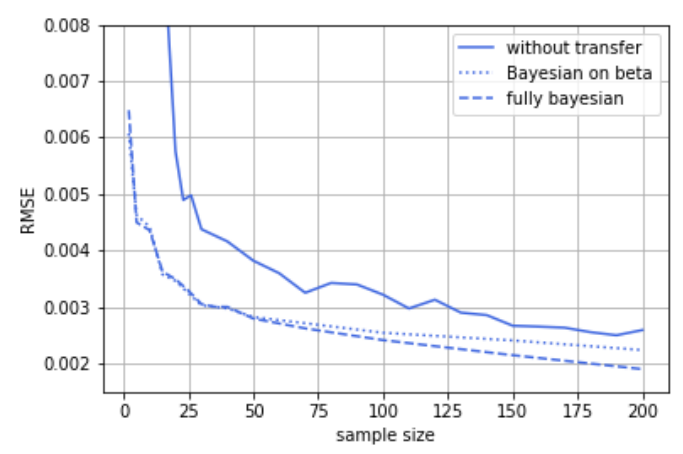

Fig. 7. Comparison of the different kriging approaches tested.

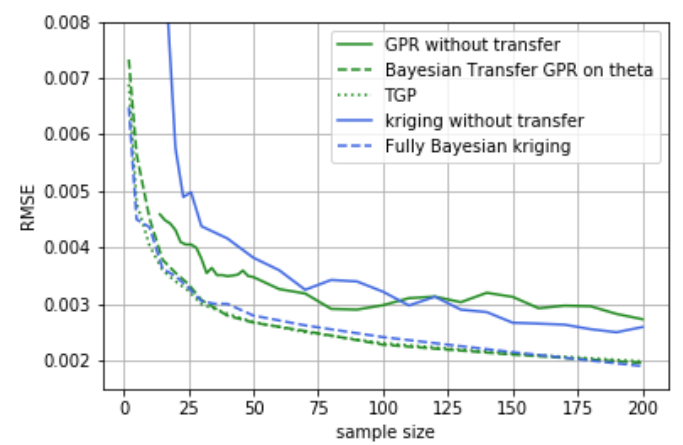

Fig. 8. Comparison of kriging and GP.

important point for the process engineers who will use the model. Therefore, the kriging model is of better interest for the industrial application under study in the present work.

\subsubsection{Conclusion for Bayesian transfer of the kriging model}

To conclude, transfer learning for the kriging method is very efficient. To obtain an RMSE score of 0.004 , one needs about 5 points instead of about 25 without transfer learning. For scores of 0.004 and 0.003 , it is 12 versus 45 and 25 versus 110 , respectively. An RMSE score of 0.0019 is obtained with 200 points, which is better than the model learned from scratch and probably not possible without transfer learning.

Concerning the computational time, the time consuming part is the calculation and the inversion of the covariance matrix which has complexity $\mathcal{O}\left(n_{t}^{3}\right)$. For the Section 3.2.1, this needs to be done only once per sample, which is quite fast with small data set and takes less than a second for one sample on a 2,6 GHz Intel Core i7 with 16 Go RAM. But for the Section 3.2.2, an MCMC algorithm is used and for a given sample, this is done for each iteration and for each modification of $\boldsymbol{\theta}_{t j}, \sigma_{t}$ and $\tau_{t}$, which means $5000 \times 14$ times in this case and takes around 20 minutes for one sample of size 200 . 


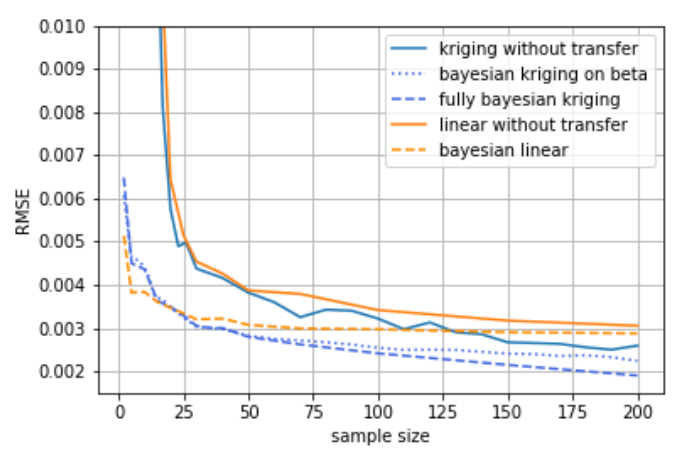

Fig. 9. Comparison of Linear Model and kriging results.

\subsection{Comparison of linear model and kriging model transfer learning}

The Bayesian approach carried out for the two models, the kriging and linear model, gives us a real improvement in terms of the number of observations needed to learn a performing model (Figure 9). With only 5 new points, the linear model transferred with the Bayesian approach give us an RMSE score that requires about 50 points for a model learned without knowledge of the source data set. This represents a cost of 50k euros instead of 500k euros. For the kriging model transferred with the Bayesian approach, 12 new points are required. For a small size of $n_{t}$, lower than 15 , the transferred linear model is slightly better than the transferred kriging model. This can be explained by the fact that the linear model is a simpler model, with fewer parameters, thus requiring less observation to be fitted. Moreover, the quality of the kriging model depends directly on the number of observations whose real values are known, since these values are directly used for the prediction. It seems that when the number of observations is lower than the number of features, the linear model performs better.

With more target points, however, the transferred kriging model improves and the gap widens when the number of points becomes larger. Our score objective is reached with 5 points and Bayesian linear model, but if one wants to get an even better score, kriging provides a solution. Moreover, even when the number of new observations is relatively large, the use of this method improves the prediction performance of the model.

\section{Conclusion and future work}

The objective was to build an efficient predictive model for a new catalyst with few new data. To do this, we used a second data set from an old catalyst for which many observations were available. Different models were tested on this second data set in order to build an efficient predictive model. Transfer learning approaches were then tested on the linear and kriging models to build the new model for the new catalyst. The Bayesian approach carried out for the two models, the kriging and linear model, gives us a real improvement in terms of the number of observations needed to learn a performing model.

The Bayesian transfer approach is therefore recommended, regardless of the number of points available. If a reduced number of points is available, less than 15 , it is advisable 
to use a simple model such as the linear model for Bayesian transfer. The RMSE score is below the target of 0.005 with only 3 new points and quickly goes below 0.004. If more points are available, it would then be advisable to use the full Bayesian kriging model, which would lead to better results. It is really efficient on our data sets due to their proximity. However, this is the industrial reality and it is a common case where we try to model a phenomenon that has changed slightly and needs to be modeled again. In our challenge, the chemical processes are the same and only the catalyst steers the reaction differently, but the features necessarily have similar influences on the reaction. If the source and target data sets are too different, this approach may not be effective.

We have applied this method for the case of diesel density prediction, but it can be applied for any type of problem for which the kriging or linear models are efficient, and therefore for any type of product quality. A possibility is to extend this Bayesian approach to kinetic models, consisting of solving a differential equation system, which are widely used to simulate the hydrocracking process (Ancheyta, Sánchez, and Rodríguez (2005); Becker, Serrand, et al. (2017); N. Y. P. Cao et al. (2020)). Further work will also focus on the design of experiments. In this paper, the data points from the target set were selected randomly. The results can certainly be improved by choosing the points to be measured to build the new model. The Kennard-Stone's algorithm (Kennard and L. Stone (1969)) is a possibility that will be explored. Other approaches such as D-optimality or A-optimality have proven their worth in experimental design (Celse, J. J. D. Costa, and V. Costa (2016); De Aguiar et al. (1995); Nikolov, Singh, and Tantipongpipat (2019)) and will be tested.

\section{References}

Ancheyta, J., S. Sánchez, and M. A. Rodríguez (2005). "Kinetic modeling of hydrocracking of heavy oil fractions: A review". In: Catalysis Today, pp. 76-92.

Argyriou, A., T. Evgeniou, and M. Pontil (2007). "Multi-task feature learning". In: Advances in neural information processing systems, pp. 41-48.

Argyriou, A., M. Pontil, et al. (2008). "A spectral regularization framework for multi-task structure learning". In: Advances in neural information processing systems, pp. 2532 .

Becker, P. J., B. Celse, et al. (2016). "A continuous lumping model for hydrocracking on a zeolite catalysts: model development and parameter identification". In: Fuel, pp. $73-82$.

Becker, P. J., N. Serrand, et al. (2017). "A single events microkinetic model for hydrocracking of vacuum gas oil". In: Computers 83 Chemical Engineering, pp. 70-79.

Bouveyron, C. and J. Jacques (2010). "Adaptive linear models for regression: improving prediction when population has changed". In: Pattern Recognition Letters, pp. 22372247.

Breiman, Leo (2001). "Random forests". In: Machine learning 45(1), pp. 5-32.

Breunig, M.M. et al. (2000). "LOF: identifying density-based local outliers". In: Proceedings of the 2000 ACM SIGMOD international conference on Management of data, pp. 93-104. 
Cao, B. et al. (2010). "Adaptive Transfer Learning". In: Proceedings of the TwentyFourth AAAI Conference on Artificial Intelligence, pp. 407-412.

Cao, N. Y. P. et al. (2020). "Accelerating Kinetic Parameter Identification by Extracting Information from Transient Data: A Hydroprocessing Study Case". In: Catalysts, p. 361.

Celse, B., J. J. D. Costa, and V. Costa (2016). "Experimental Design in Nonlinear Case Applied to Hydrocracking Model: How Many Points Do We Need and Which Ones?" In: International Journal of Chemical Kinetics, pp. 660-670.

Cressie, N. (1988). "Spatial prediction and ordinary kriging". In: Mathematical geology, pp. 405-421.

Cressie, N. (1990). "The origins of kriging". In: Mathematical geology, pp. 239-252.

Da, Bingshui et al. (2019). "Fast transfer Gaussian process regression with large-scale sources". In: Knowledge-Based Systems 165, pp. 208-218.

Dai, W. et al. (2007). "Boosting for Transfer Learning". In: Proceedings of the 24th international conference on Machine learning, pp. 193-200.

De Aguiar, P. F. et al. (1995). "D-optimal designs". In: Chemometrics and intelligent laboratory systems 30, pp. 199-210.

Drucker, H. et al. (1996). "Support vector regression machines". In: Advances in neural information processing systems, pp. 155-161.

Evgeniou, T. and M. Pontil (2004). "Regularized multi-task learning". In: Proceedings of the tenth ACM SIGKDD international conference on Knowledge discovery and data mining, pp. 109-117.

Friedman, J. H. (2001). "Greedy function approximation: a gradient boosting machine". In: Annals of statistics 29, pp. 1189-1232.

Ginsbourger, David et al. (2009). "A note on the choice and the estimation of kriging models for the analysis of deterministic computer experiments". In: Applied Stochastic Models in Business and Industry 25(2), pp. 115-131.

Helbert, C., D. Dupuy, and L. Carraro (2009). "Assessment of uncertainty in computer experiments from Universal to Bayesian Kriging". In: Applied Stochastic Models in Business and Industry, pp. 99-113.

Kennard, R.W. and L.A. Stone (1969). "Computer aided design of experiments". In: Technometrics, pp. 137-148.

Launay, T., A. Philippe, and S. Lamarche (2015). "Construction of an informative hierarchical prior for a small sample with the help of historical data and application to electricity load forecasting". In: Test, pp. 361-385.

Liu, Haitao, Jianfei Cai, and Yew-Soon Ong (2018). "Remarks on multi-output Gaussian process regression". In: Knowledge-Based Systems 144, pp. 102-121.

Nikolov, A., M. Singh, and U. T. Tantipongpipat (2019). "Proportional volume sampling and approximation algorithms for A-optimal design". In: Proceedings of the Thirtieth Annual ACM-SIAM Symposium on Discrete Algorithms. Society for Industrial and Applied Mathematics, pp. 1369-1386.

Pan, S. and Q. Yang (2010). "A Survey on Transfer Learning". In: IEEE Transactions on Knowledge and Data Engineering, pp. 1345-1359.

Pardoe, D. and P. Stone (2010). "Boosting for Regression Transfer". In: Proceedings of the 27th international conference on Machine learning, pp. 863-870. 
Pedregosa, F. et al. (2011). "Scikit-learn: Machine Learning in Python". In: Journal of Machine Learning Research 12, pp. 2825-2830.

Raina, R., A. Y. Ng, and D. Koller (2006). "Constructing informative priors using transfer learning". In: Proceedings of the 23rd international conference on Machine learning, pp. 713-720.

Rasmussen, Carl Edward and Christopher K.I. Williams (2006). Gaussian processes formachine learning. Taylor \& Francis Group.

Roustant, O., D. Ginsbourger, and Y. Deville (2012). "Dicekriging, Diceoptim: Two R packages for the analysis of computer experiments by kriging-based metamodelling and optimization". In: Journal of Statistical Software, 54p.

Rumelhart, David E, Geoffrey E Hinton, and Ronald J Williams (1985). Learning internal representations by error propagation. Tech. rep. California Univ San Diego La Jolla Inst for Cognitive Science.

Salaken, S. M. et al. (2019). "Seeded transfer learning for regression problems with deep learning". In: Expert Systems with Applications, pp. 565-577.

Seber, George AF and Alan J Lee (2012). Linear regression analysis. Vol. 329. John Wiley \& Sons.

Segev, N. et al. (2016). "Learn on source, refine on target: a model transfer learning framework with random forests". In: IEEE Transactions on pattern analysis and machine intelligence, pp. 1811-1824.

Stein, M. L. (2012). Interpolation of spatial data: some theory for kriging. Springer Science \& Business Media.

Tierney, L. (1994). "Markov chains for exploring posterior distributions". In: the Annals of Statistics, pp. 1701-1728.

Tsung, F. et al. (2018). "Statistical transfer learning: A review and some extensions to statistical process control". In: Quality Engineering, pp. 115-128.

Wei, Pengfei et al. (2017). "Source-target similarity modelings for multi-source transfer gaussian process regression". In: International conference on machine learning. PMLR, pp. 3722-3731.

Zellner, A. (1986). "On assessing prior distributions and Bayesian regression analysis with g-prior distributions". In: Goel, P. and Zellner, A., Eds., Bayesian Inference and Decision Techniques: Essays in Honor of Bruno de Finetti, Elsevier Science Publishers, pp. 233-243. 